\title{
A Survey on Assorted Subsisting Approaches to Recognize and Preclude Black Hole Attacks in Mobile Adhoc Networks
}

\author{
https://doi.org/10.3991/ijim.v14i01.11329 \\ M. Thebiga $\left({ }^{\varpi}\right)$, R. Suji Pramila \\ Noorul Islam Centre for Higher Education, Kumaracoil, TamilNadu \\ chikka2001@gmail.com
}

\begin{abstract}
Ensuring collateral is the furthest substantial snag in Mobile Adhoc Networks which crash the efficacy of the network. Without regard to all different networks, the Mobile Adhoc network is stuffed with more safety hindrances and the Adhoc on Demand Vector Routing Protocol is more comprehensively utilized customs in MANETS. This type of network is more exposed to assorted number of attacks and among those, the black hole attack and its variant pull off critical detriment to the entire network .In this type of attack, named black hole attack, the noxious node utilizes its routing principles, with the view to annunciate itself, that it has the briefest route to the target node. In this research paper, we have explored all the subsisting techniques and graded the solution with a table to understand their pros and cons.
\end{abstract}

Keywords-Mobile Adhoc Networks, Adhoc on Demand Routing Protocol, Networks, Attacks, Black Hole Attacks, Noxious node.

\section{Introduction}

Mobile adhoc networks is the special type of networks, which can be explicated as a throng of wireless devices that has the potential of dynamically varying topology [1]. Because of very good expansion in wireless networking methodology, the mobile adhoc network is procuring more consideration in different types of applications such as entertainment, VANETs, emergency handling, sensor networks, military applications etc. [2]. Owing to the openness nature and ever changing configuration, and as a consequence of some quirky features such as bandwidth impediment, computation power damper, infrastructure less networks etc., the mobile adhoc networks are exposed to distinct categories of attacks [3].The attacks facing to Mobile adhoc networks can be assorted into two groups on the grounds of basic characters. They are:

- Active Attacks

- Passive Attacks [4]

An active attack is a breed of attack, in which it endeavors to amend the system assets or it strives to disrupt the entire system performance. Active attacks entails cer- 
tain mutation in the data patterns. Some categories of active attacks are impersonation, mutation, disavowal, and Denial of service attacks. Passive attacks

Is a breed of attack in which it entails to discover or take advantage of the network information from the system, however it fails to influence the system assets. The main intention of this attack is to procure information from the competitor. The examples of passive attacks are launching message, examining the traffic. On the grounds of domain, the vulnerable attacks facing in MANETs can be assorted into two groups. They are:

- Internal Attacks

- External Attacks

External Attacks is a breed of attacks, in which the attacks are put into effect by the nodes that do not adhere to the scope of this sort of network. Inward attacks are conducted by the nodes in which these sort of nodes are actually endangered, and it adhere to the scope of network. [5]. Offering security or reliability in Mobile adhoc network for the purpose to mail the data from initiator node to target node, is actually a complicated and daring task. Multitudinous research investigations are carried out to enhance the security of the Mobile Adhoc Networks. In general, most of the explorations cope with perception and preclusion techniques to counter against hostile node. When the potency of those procedures turns into feeble, then numerous hostile nodes blend mutually to trigger a synergistic attack, which causes a severe detriment to the system network [6].Inadequate substructure along with dynamic changing topology qualities of Mobile Adhoc Networks fabricate this type of networks to face distinct varieties of security attacks such like black hole attack, Refutation of service attack, and grey hole attacks. Black hole attack is a breed of attack, whereupon hostile nodes captivates the data chunks by fallaciously heralding a new and latest route to the target node. In this recommended paper, we emphasize on the AODV (Adhoc on Demand Routing Protocol) type Protocol, Black Hole type of Attacks, and different methodologies that has been suggested to discover and to evade black hole type of attacks in mobile type of adhoc networks are discussed and compared in a table.

\section{Black Hole Attacks in MANETs}

Black Hole Attacks in mobile adhoc network is a sort of active attack, in which the vicious node publicize that it comprises the quickest track to the final node, even though it fails to have path to the target node. In view of this, each and every packets will move through the vicious node and this will permits that hostile nodes to either broadcast the packet or drip the packet. The initiator node circulates a Route Request packet to the target node. In this circulation, the node whoever acquires this route request packet audits whether this particular node has the route track to the target node. When this vicious node acquires the route requesting message, it promptly respond back to the initiator node, informing that it comprises the recent and soonest route track to the target node. Initiator node entrust that response from the neighbor node, and starts relaying Those packets to the vicious node, expecting that this partic- 
ular node will dispatch the message packet successfully to the target node [7]. The initiator node does not uses any methods or techniques to audit whether that reply from the node is genuine or not.

After receiving the packet the vicious node starts to either forward the packet or drip all packets and creates black hole attack in MANETs.

Table 1. Attacks in Various strata's

\begin{tabular}{|c|l|l|}
\hline SI.No & \multicolumn{1}{|c|}{ Different Strata's } & \multicolumn{1}{c|}{ Attacks } \\
\hline 1 & Physical Strata & (i)Isolate and prohibits Jamming attacks \\
\hline 2 & Data Link strata & $\begin{array}{l}\text { (i)Active attacks such as attacks in IEEE 802.11 } \\
\text { Medium Access Control Layers. } \\
\text { (ii)Passive attacks such as traffic surveillance } \\
\text { and examination, snooping }\end{array}$ \\
\hline 3 & Network Strata & $\begin{array}{l}\text { (i)Active attacks such as Black hole type attack, } \\
\text { Grey Hole type attack, Packet dripping type } \\
\text { Attack, Man in the Middle type attack, Sleep } \\
\text { Loss attack, Discontinuity attack, and } \\
\text { Swamping attack. } \\
\text { (ii)Passive sort of attacks such as Position Dis- } \\
\text { Covery attack. }\end{array}$ \\
\hline 4 & Transport Strata & $\begin{array}{l}\text { (i)Active attacks such as Event Abduction } \\
\text { Attack, Change by reversal attack. }\end{array}$ \\
\hline 5 & Application Strata. & $\begin{array}{l}\text { (i)Repudiation of service attack, Disavowal } \\
\text { Attack etc. }\end{array}$ \\
\hline
\end{tabular}

\section{Black Hole Attacks in MANETs}

Black Hole Attacks in mobile adhoc network is a sort of active attack, in which the vicious node publicize that it comprises the quickest track to the final node, even though it fails to have path to the target node. In view of this, each and every packets will move through the vicious node and this will permits that hostile nodes to either broadcast the packet or drip the packet. The initiator node circulates a Route Request packet to the target node. In this circulation, the node whoever acquires this route request packet audits whether this particular node has the route track to the target node. When this

Vicious node acquires the route requesting message, it promptly respond back to the initiator node, informing that it comprises the recent and soonest route track to the target node. Initiator node entrust that response from the neighbor node, and starts relaying Those packets to the vicious node, expecting that this particular node will dispatch the message packet successfully to the target node [7]. The initiator node does not uses any methods or techniques to audit whether that reply from the node is genuine or not. After receiving the packet the vicious node starts to either forward the packet or drip all packets and creates black hole attack in MANETs. 


\section{$4 \quad$ Related Works}

\subsection{Modified AODV algorithm}

Sin et.al [8] recommended a latest procedures to face black hole attacks. This procedure isolate the vicious node in consonance with the nodes features and wiped out from the network. In this technique, they have suggested some set of regulation for the discovery of viscous nodes. The regulations for the discovery of vicious nodes are nodes which replies Route Reply to the initiator node which sends Route Request, the nodes with minimal number of hops, higher succession number, the nodes which broadcast maximum count of data chunks, and the node which acquires maximum count of data chunks and dispatches minimum count of data chunks. Only with these regulation, we are not able to affirm that specific node is vicious node.

\subsection{Modified DSR protocol algorithm}

Y Mohana Priya et.al [9] suggested a newly revised Dynamic source Routing protocol to disclose and to hinder the vicious node by means of incursion detection method

If the likelihood of count of data chunks acquired is higher than the preset threshold value, then the target node initiates the discovery of vicious node. In terms of vicious node discovery process, the. destination node send a Query Routing request (QROREQ) to the two skip count node and check the count of packets relayed .If the count of packets relayed are equalized, then reiterate the procedure for the next two skip count node,else, include the information of two skip nodes and the subsequent node into the dubious list. If the variance between the counts of packets relayed between the two suspected nodes goes beyond the preset threshold value, then that individual nodes are affirmed as vicious node. Once the vicious nodes are discovered, the information about the specific nodes are circulated to the closest Incursion Detection System. The Incursion detection system will track the dubious nodes. If that dubious nodes drips the

Packet, then that individual node is affirmed as vicious node, and that individual node

Is secluded from the routing process and the information about that vicious node is Circulated to every node. This method has maximum message routing overhead on account of conveyance of Query Routing Request, Query Routing Reply, Vicious node Route Request, and alarm data packets.

\subsection{Packet loss analysis technique}

Tao et.al [10] recommended a new procedure to employ the association between the dripped data chunks which is reckoned by means of Auto Correlation function of dropped data chunks bitmap mode. By discovering the association in between the dripped data chunks, we can observe the packet drip as a consequence of either link 
error or malevolent drop. To authenticate the veracity of recorded information, a publicly monitoring technique is employed which is purely in reference to homomorphic Linear authenticator enciphering methods. In this method, for each transmission of data chunks, HLA signatures are generated. Origination of HLA Signatures for each transmission is considered as a very expensive technique. This method is restrained to stagnant and apparently semi stagnant wireless adhoc networks. More number of encryption, decryption, message authentication code and hashing techniques are employed for the discovery of vicious node and for the secured data transmission between the nodes. This method cannot be implemented for all types of routing protocols.

\subsection{Novel based honey pot method}

Rajesh et.al [12] recommended a new honey pot technique to trap the vicious node. To initiate this honey pot trap method, a spoofed routing request is circulated to the nearest node. The node which accepts this spoofed request and send back a routing reply packet which indicates that it has the route to the target node, will affirmed as the vicious node. The spoofed request node holds two domains, one is unavailable identi-

Fier of the node, instead of succession number domain, and the second one is time to live domain. The vicious node is isolated and the knowledge about that hostile node is circulated to every else node in the connected network. The cons behind this method is

Instead of using succession number, the nonavailablility node id is employed. Normally the succession number domain is used to avoid musty routes. By avoiding the use of succession number, the number of musty routes will be increased.

\subsection{Erratic two skip count ACK and bayesian perception method}

Djenouri et.al [13] suggested a new erratic two skip count confirmatory technique and Bayesian Perception method to discover and insulate the black hole attack in Mobile Adhoc Networks. In the monitoring phase, each node will check its two hop neigh-bor, whether it forwards the data correctly, if so a positive two skip acknowledgment is send back to the source. The process is repeated until reaches the target node. A Bayesian based technique is used to judge whether the particular node is vicious node or honest node. In the Bayesian based approach method, if a node is affirmed as malevolent node, then a proper adjudication should be confirmed by all other nodes involved in the network. This method does not have a frequent swap of messages which results in less routing overhead. The author also recommended a witness based approach that pressurize the perceived node to assure the judgement given by different nodes involved in the network. Prior to the seclusion of vicious node, witness approach pressurize the discoverer to accumulate at the very least of ' $n$ ' witnesses. Maximum number of $n$ values of witnesses diminishes the detection accuracy. Simulation results proves that this suggested work obtains a reduced falsely detection rate and increased truly detection Rate, in compared to existing work. The 
cons behind this work is, it is very much challenging one to discover the collaborative black hole attack, if certain vicious node betrays the perceived node co-actively.

\subsection{Neighbor collaborative bait detection scheme}

Jian [14] et.al recommended a new perception method titled as collaborative bait approach to observe and to hinder the black hole and greyhole attacks in Mobile Adhoc Networks. In this work, the initiator hypothetically elect the proximate nodes address as the trap address to trick the vicious node to forward a response message. By the way of revoke tracking method, the vicious nodes are observed and hindered from the normal routing procedure. If there is serious packet drip, an alarm packet is forwarded from the target node to the initiator, to provoke the identification procedure. The information of the observed vicious nodes is stored in blacklist and that information is circulated to every other nodes in the network, and finally that Particular node id is segregated from the network. This method consolidate the strengths of both table motivated routing protocol and on request routing protocol which diminishes the squandering of resources. The simulation results proves that in this method, when the percentage of vicious nodes

is fixed, it gives a diminished packet delivered ratio, increased convoying overhead, diminished Throughput and increased end to end delay, when the nodes mobility rises.

\subsection{Detection based on dynamic threshold value}

Raj et.al [15] nominated a creative method which is based on energetic threshold value to discover the black hole attack in Manets. The suggested method is different from normal AODV protocol. Apart from this, a supplemental verification is done, i.e., whenever the node assents the Route Replying packet, it will verify whether the route reply succession number goes beyond the preset threshold value. If so, that particular node is doubted as vicious node and recorded in black list. So an ALARM packet encloses the black list node identifier as a parameter, is generated and circulated through-

Out the network .so if any node assents the route reply, it will verify whether the response is from black listed node. If so, it will discard the reply packet. Dynamically changing boundary value is estimated by reckoning the mean value of succession number and the reply packets. By means of this technique, the black hole attack is hindered.

Simulation results proves that it has an increased end to end delay and routing overhead.

\subsection{Composite intrusion detection system}

Lauf et.al [16] suggested a new composite Intrusion Detection technique to discover the vicious node. This composite IDS comprises three phases. First one is Maximum discovery training phase, second one is cross association discovery training 
phase, and the third one is Cross association discovery training phase. Based upon the interactivity between the nodes in the application level, a standard portrait is created during the training phase. The Maximum discovery system preserves the records of the application level interactivity between the nodes. In the tracking phase, the standard profile is equalized with the records of the interactivity to observe possible deviations. Maximum Discover system discovers possible menaces and computes a proper threshold value to observe the root causes of single attacks or numerous attacks concurrently. This method provides maximum false positive rates and the diminished detection rate, because of incongruous computation of threshold value.

\subsection{Game theory based technique}

Rafsanjani et.al [18] suggested a new technique to avert inward attacker and discover outward attacker in mobile adhoc networks by applying game theory concepts. The propounded concept has three phases. First phase is, to avert inward attack, by employ-

Ing the Bayesian concept, trusted value for every node in the clusters are estimated. If the adjacent nodes calculated trusted value is lower than the preset boundary value, then that individual node is labelled as vicious node. In the second level, the node with adequate energy level will be chosen as cluster head. In the third phase, outward attackers are discovered by applying Bayesian game theory concepts. Threshold values are estimated to report the suffered node to initiate the Incursion discover system, when the likelihood of attack goes beyond the predefined threshold value. Because of employing Bayesian game theory concept and electing a trustful cluster head, the reliability and the efficiency of the network was expanded. The drawback behind this approach is, there is no proper investigations has been done to the nodes mobility in case of detecting vicious nodes.

\subsection{Trust based approach}

Weizhi et.al [19] suggested a new packet filtering technique to protect the network from attacks. In this work, they have configured a synergistic belief dependent packet filtering method, which successfully diminishes the traffic in case of synergistic environment. Bayesian concepts are employed for the estimation of trust in the configuration of packet filter, which ultimately diminishes the undesirable packets and the burden of work in Incursion Discover system. To upgrade the permanence and the presentation of the network, synergistic belief based packet filter was configured to estimate the trust value for a node and an IP initiator, by assembling all intelligence and knowledge information. IP assurance indicates the reliability of an IP initiator. The organization of synergistic belief dependent packet filter comprises three elements. First one is synergistic elements, second one is trust estimation element, and third one is black listed Packet filtration element. In the synergistic compartment, all the information about the nodes are gathered and relayed the necessary information to the second compartment Named Trust estimation, in which the trust values are estimated .The black listed compartment will filter the necessary packets based on IP 
assurance value. It comprises all suspicious IP addresses. The achievement of this proposed packet filter is examined in the presence of truthful conditions and deceptive conditions. The proposed packet filter with honesty trust estimation was recognized as a more stable one to face deception attacks. The drawback behind this proposed work is, byzantine attacks cannot be observed using this packet filter. This work creates a database issue, as lot of signatures and volume of look up table got expanded.

Antesar et.al [21] suggested a new suggestions dependent trust pattern to percolate attacks connected to false suggestions such like bad muttering attack, complicity attack in mobile adhoc networks. The suggested node is elected in accordance with three contexts. First one is the count of correlation between the judged nodes, integrity opinion, and proximity with the judging node. The author employed a Bayesian method to estimate the trust values which depends upon two criteria such like relayed packets and dripped packets. This model comprises three elements, trust estimation element, recommendation filter, and third one is cluster formation element. The trust estimation element estimates trust value by means of direct trust. The recommendation filter element assist in observing and first it will send the suggestion requisition to the nearest nodes, and second the acquired suggestion clustered based on three criteria's, confidence range, deviation range and Euclidean distance between nodes, and the third function is acquired screened suggestion $\mathrm{s}$ are given as input to the trust estimation element, and finally trusted clusters are received. The drawback behind this work is high memory

Utilization .Simulations results shows that this propounded security mechanisms Includes proper deviant trust evidences acquired by suggestions and eradicate dishonest Nodes.

Danyang et.al [22] suggested a new lightweight trust based method to defy more Number of attacks in Wireless networks. Direct trust is estimated by means of communication Behavior and energy level trust. Indirect trust is calculated using deviant Behavior trust and deviant energy trust. A secured routing path is chosen by means of

Calculated trust values and some Quality of service criteria's and by employing semiring theory. Simulation results proves that this proposed work will diminishes message routing overhead and enhance the security of forwarding packets from the initiator node to the destination. 
Table 2. Comparative Investigations on present Approaches

\begin{tabular}{|c|l|l|l|l|}
\hline Sl.no & \multicolumn{1}{|c|}{ Authors Name } & \multicolumn{1}{c|}{ Approaches } & \multicolumn{1}{c|}{ Merits } & \multicolumn{1}{c|}{ Constraints } \\
\hline 1. & $\begin{array}{l}\text { Sina Shahabi, Mah- } \\
\text { dieh Ghazvini,et.al [8] }\end{array}$ & $\begin{array}{l}\text { (i) Set of regulations to } \\
\text { recognize viscous node. }\end{array}$ & $\begin{array}{l}\text { (i)Reduced Packet } \\
\text { drip rate } \\
\text { (ii)Good Through- } \\
\text { put }\end{array}$ & $\begin{array}{l}\text { (i)With these set of } \\
\text { regulations, we cannot } \\
\text { affirm the node as } \\
\text { vicious node }\end{array}$ \\
\hline 2. & $\begin{array}{l}\text { M.MohanaPriya, } \\
\text { Illango Krishnamurthi, } \\
{[9]}\end{array}$ & $\begin{array}{l}\text { (i)Use of Incursion } \\
\text { Discover Method } \\
\text { (ii)forward Query Rout- } \\
\text { ing Request to two skip } \\
\text { count node }\end{array}$ & $\begin{array}{l}\text { (i)Minimized Packet } \\
\text { Drip rate } \\
\text { (ii)Diminished End } \\
\text { to End Delay }\end{array}$ & $\begin{array}{l}\text { (i)Everged Routing } \\
\text { overhead } \\
\text { (ii)When the count of } \\
\text { IDS diminishes, the } \\
\text { PDR value got re- } \\
\text { duced. }\end{array}$ \\
\hline Krunz, [10] & $\begin{array}{l}\text { (i)Association between } \\
\text { Dripped Packet } \\
\text { (ii)Public Auditing by } \\
\text { HLA enciphering tech- } \\
\text { nique. }\end{array}$ & $\begin{array}{l}\text { (i) Very Good } \\
\text { (ii)Less conveyance } \\
\text { overhead }\end{array}$ & $\begin{array}{l}\text { (i)Origination of HLA } \\
\text { signatures is extor- } \\
\text { tionate. } \\
\text { (ii)Restrained to } \\
\text { stagnant and apparent- } \\
\text { ly semi stagnant } \\
\text { wireless adhoc net- } \\
\text { works } \\
\text { (iii)Cannot be imple- } \\
\text { mented for all types of } \\
\text { routing protocols }\end{array}$ \\
\hline
\end{tabular}

Table 3. Comparative Investigations on present Approaches

\begin{tabular}{|c|l|l|l|l|}
\hline Sl.no & \multicolumn{1}{|c|}{ Authors Name } & \multicolumn{1}{c|}{ Approaches } & \multicolumn{1}{c|}{ Merits } & \multicolumn{1}{c|}{ Constraints } \\
\hline 1. & $\begin{array}{l}\text { Muhammad Saleem, } \\
\text { Daniele Midi, Majid } \\
\text { Iqbal khan, Elisa } \\
\text { Bertino [11] }\end{array}$ & $\begin{array}{l}\text { (i) Parameters that are } \\
\text { used for the recognition } \\
\text { of packet drip are Medi- } \\
\text { um Access Control layer } \\
\text { data's, relaying nodes } \\
\text { queuing data's, and } \\
\text { finally the association } \\
\text { between nodes infor- } \\
\text { mation }\end{array}$ & $\begin{array}{l}\text { (i)Increased Detec- } \\
\text { tion rate }\end{array}$ & $\begin{array}{l}\text { (i) Cannot be worked } \\
\text { out in other routing } \\
\text { protocol } \\
\text { (ii)Higher Energy } \\
\text { utilization }\end{array}$ \\
\hline 2. & $\begin{array}{l}\text { M.Rajesh Babu, } \\
\text { G.Usha [12] }\end{array}$ & $\begin{array}{l}\text { (i) Honey pot technique } \\
\text { to trap the vicious node } \\
\text { (ii)Spoofed routing } \\
\text { request is circulated }\end{array}$ & $\begin{array}{l}\text { (i)Less packet drip } \\
\text { (ii)PDR is 89\% }\end{array}$ & $\begin{array}{l}\text { (i)Non-availability } \\
\text { node id is employed } \\
\text { (ii) The number of } \\
\text { musty routes will be } \\
\text { increased. }\end{array}$ \\
\hline 3. & $\begin{array}{l}\text { Djenouri D, Badache } \\
\text { [13] }\end{array}$ & $\begin{array}{l}\text { (i)Two skip count con- } \\
\text { firmatory technique and } \\
\text { Bayesian Perception } \\
\text { method }\end{array}$ & $\begin{array}{l}\text { (i)Reduced false } \\
\text { detection and in- } \\
\text { creased true detec- } \\
\text { tion rate }\end{array}$ & $\begin{array}{l}\text { (i)Maximum number } \\
\text { of n values of witness- } \\
\text { es diminishes the } \\
\text { detection accuracy. } \\
\text { (ii)Challenging one to } \\
\text { recognize the collabo- } \\
\text { rative black hole } \\
\text { attack. }\end{array}$ \\
\hline
\end{tabular}


Table 4. Comparative Investigations on present Approaches

\begin{tabular}{|c|l|l|l|l|}
\hline Sl.no & \multicolumn{1}{|c|}{ Authors Name } & \multicolumn{1}{c|}{ Approaches } & \multicolumn{1}{c|}{ Merits } & \multicolumn{1}{c|}{ Constraints } \\
\hline 1. & $\begin{array}{l}\text { Jian-MingChang,Po, } \\
\text { Po-Chun Tsou,Isac } \\
\text { Woungang, Han- } \\
\text { Chieh Chao, and Chin- } \\
\text { Feng Lai [14] }\end{array}$ & $\begin{array}{l}\text { (i) Collaborative bait } \\
\text { approach } \\
\text { (ii)Choose the nearby } \\
\text { nodes address as the trap } \\
\text { address to trick the vi- } \\
\text { cious node }\end{array}$ & $\begin{array}{l}\text { (i) Consolidate the } \\
\text { strengths of both } \\
\text { table driven routing } \\
\text { protocol and on } \\
\text { demand routing } \\
\text { protocol }\end{array}$ & $\begin{array}{l}\text { (i) diminished packet } \\
\text { delivery ratio, when } \\
\text { the percentage of } \\
\text { vicious nodes is fixed } \\
\text { (ii) It demands an } \\
\text { increased time to } \\
\text { observe a vicious node }\end{array}$ \\
\hline 2. & Raj P.N, Swadas [15] & $\begin{array}{l}\text { (i)Method based on } \\
\text { energetic threshold value } \\
\text { (ii)Take average value of } \\
\text { succession number and } \\
\text { the reply packets }\end{array}$ & $\begin{array}{l}\text { (i) Prevents Black- } \\
\text { hole attack }\end{array}$ & $\begin{array}{l}\text { (i) Increased end to } \\
\text { end delay and routing } \\
\text { overhead }\end{array}$ \\
\hline 2. & $\begin{array}{l}\text { (i)Composite Intrusion } \\
\text { Retection technique } \\
\text { (ii)Three phases } \\
\text { (a)Maximum discovery } \\
\text { training phase, } \\
\text { (b)cross association } \\
\text { discovery training phase, } \\
\text { (c)Cross association } \\
\text { discovery training phase }\end{array}$ & $\begin{array}{l}\text { (i) Discovers possi- } \\
\text { ble menaces }\end{array}$ & $\begin{array}{l}\text { (i)Maximum false } \\
\text { positive rates and the } \\
\text { diminished detection } \\
\text { rate }\end{array}$ \\
\hline
\end{tabular}

Table 5. Comparative Investigations on present Approaches

\begin{tabular}{|c|c|c|c|c|}
\hline Sl.no & Authors Name & Approaches & Merits & Constraints \\
\hline 1. & $\begin{array}{l}\text { Nadeem A, Howarth } \\
\text { MP, [17] }\end{array}$ & $\begin{array}{l}\text { (i) Blends the statistical } \\
\text { based and knowledge } \\
\text { based Intrusion detection } \\
\text { technique } \\
\text { (ii)network feature ma- } \\
\text { trix, network operational } \\
\text { matrix are employed }\end{array}$ & $\begin{array}{l}\text { (i) Good detection } \\
\text { rate }\end{array}$ & $\begin{array}{l}\text { (i) Discovery of any } \\
\text { abnormalities can be } \\
\text { carried out only by the } \\
\text { Manager node } \\
\text { (ii) Huge collection of } \\
\text { information and } \\
\text { increased processing } \\
\text { time. }\end{array}$ \\
\hline 2. & $\begin{array}{l}\text { Rafsanjani MK, } \\
\text { Aliahmadipour L, } \\
\text { Javidi MM [18] }\end{array}$ & $\begin{array}{l}\text { (i) Apply game theory } \\
\text { concepts } \\
\text { (ii)Three phases } \\
\text { a)Apply Bayesian con- } \\
\text { cept to estimate trust } \\
\text { value, to avert inward } \\
\text { attacker } \\
\text { b)outward attackers are } \\
\text { discovered by applying } \\
\text { Bayesian game theory } \\
\text { concepts }\end{array}$ & $\begin{array}{l}\text { (i)Maximum relia- } \\
\text { bility and the effi- } \\
\text { ciency of the net- } \\
\text { work }\end{array}$ & $\begin{array}{l}\text { (i)No proper investiga- } \\
\text { tions has been done to } \\
\text { the nodes mobility in } \\
\text { case of detecting } \\
\text { vicious nodes }\end{array}$ \\
\hline 3. & $\begin{array}{l}\text { Weizhi Meng, } \\
\text { WenJuan, Lam For } \\
\text { Kwok [19] }\end{array}$ & $\begin{array}{l}\text { (i)Configured a synergis- } \\
\text { tic belief dependent } \\
\text { packet filtering method } \\
\text { (ii)Bayesian concepts are } \\
\text { employed for the estima- } \\
\text { tion of trust }\end{array}$ & $\begin{array}{l}\text { (i)More stable one } \\
\text { to face deception } \\
\text { attacks }\end{array}$ & $\begin{array}{l}\text { (i) Byzantine attacks } \\
\text { cannot be observed } \\
\text { (ii) Creates a database } \\
\text { issue }\end{array}$ \\
\hline
\end{tabular}


Table 6. Comparative Investigations on present Approaches

\begin{tabular}{|c|c|c|c|c|}
\hline Sl.no & Authors Name & Approaches & Merits & Constraints \\
\hline 1. & $\begin{array}{l}\text { Jinfang Jiang } \\
\text {,Guangjie Han,Feng } \\
\text { Wang,Lei } \\
\text { Shu,Mohsen Guizani, } \\
{[20]}\end{array}$ & $\begin{array}{l}\text { (i)Effectual trust depend- } \\
\text { ent paradigm }\end{array}$ & $\begin{array}{l}\text { (i) Assess the relia- } \\
\text { bility more accu- } \\
\text { rately }\end{array}$ & $\begin{array}{l}\text { (i) When the count of } \\
\text { vicious node increases, } \\
\text { the detection rate } \\
\text { diminishes. }\end{array}$ \\
\hline 2. & $\begin{array}{l}\text { Antesar M.Shabut, } \\
\text { Keshav P.Dahal, Sanat } \\
\text { Kumar Bista, Irfan } \\
\text { U.Awan [21] }\end{array}$ & $\begin{array}{l}\text { (i)Suggestions dependent } \\
\text { trust pattern. } \\
\text { (ii) Suggested node is } \\
\text { elected in accordance } \\
\text { with three contexts }\end{array}$ & $\begin{array}{l}\text { (i)Eradicate dishon- } \\
\text { est nodes. }\end{array}$ & $\begin{array}{l}\text { (i)High memory } \\
\text { utilization }\end{array}$ \\
\hline 3. & $\begin{array}{l}\text { Danyang Qin ,Shuang } \\
\text { Jia, Jingya Ma,Yan } \\
\text { Zhnag,Qun Ding [22] }\end{array}$ & $\begin{array}{l}\text { (i) Direct trust is estimat- } \\
\text { ed by communication } \\
\text { Behavior and energy } \\
\text { level trust. } \\
\text { (ii)Indirect trust is calcu- } \\
\text { lated using deviant Be- } \\
\text { havior trust and deviant } \\
\text { energy trust }\end{array}$ & $\begin{array}{l}\text { (i) Diminishes } \\
\text { message routing } \\
\text { overhead } \\
\text { (ii) Enhance the } \\
\text { security }\end{array}$ & $\begin{array}{l}\text { (i)No efficient method } \\
\text { to select Cluster head }\end{array}$ \\
\hline
\end{tabular}

\section{Conclusion and Future Work}

Mobile adhoc networks is the most momentous role in advanced communication system. Because of some unique features like infrastructure less, non-distributed system, dynamically changing network, this mobile adhoc networks is fragile to different attacks, such like Black hole attacks. Protecting the data against those attacks is very challenging task in this network. In this paper, we have surveyed all existing methods that have been propounded to recognize and avoid black hole attack in MANETs and levelled the resolution with a table to understand their ups and downs. In most of the subsisting techniques, enciphering and hashing methods are applied to recognize the vicious node, which is considered to be more exorbitant and demands additional resources. As a future work, here we conclude and propose that trust based approach will be more effective to recognize and preclude Blackhole attacks in MANETs and we entrust that this review paper will be more exquisite and very striking subject for future investigation on securities in Mobile adhoc networks.

\section{References}

[1] Murthy CSR, Manoj B.S (2012) Adhoc Wireless Networks: Architectures and Protocols, Pearson Education, Delhi, India.

[2] Hoebeke J, Moernman I, Dhoedt B, Demeester (2004) An Overview of Mobile Adhoc Networks: Applications and Challenges, Communications Network Journal, 3(3), pp 6066.

[3] Mishra. A, Nadkarni k.M (2003),'Security in wireless adhoc Networks, In the Handbook of Adhoc Wireless Networks, CRC Press: Boca Raton, FL, USA, pp 499-549. 
[4] Djenouri D, Khelladi Baadache N (2005), Security issues of Mobile Adhoc and Sensor Networks, IEEE Communications Surveys \& Tutorials 7(4), pp 2-28. https://doi.org/10.11 $\underline{09 / \text { comst.2005.1593277 }}$

[5] Wu .B, Chen J, Wu J, Cardei M (2007), A Survey of Attacks and Countermeasures in Mobile Adhoc Networks, Wireless Network Security. Springer US, pp 103-135. https:// doi.org/10.1007/978-0-387-33112-6_5

[6] Jian Ming Chang,Po Chun Tsou,Isac Woungang ,Han Chieh Chao,Chn Feng Lai(2015),Defending Against Collaborative Attacks by Malicious nodes in MANETs: A Cooperative Bait Detection Approach",IEEE Systems Journal ,9(1)Mar 2015,pp 65-75. https://doi.org/10.1109/jsyst.2013.2296197

[7] Adwan Yasin, Mahmoud Abu Zant (2018), Detecting and Isolating Blackhole Attack in Manets using a Timer based Baited Technique, Wireless Communication and Mobile Computing, Hindawi, 2018, https://doi.org/10.1155/2018/9812135.

[8] Sina Shahabi, Mahdieh Ghazvini, Mehdi Bakhtiarian(2016),A Modified Algorithm to improve security and performance of AODV protocol against Black hole Attack, Wireless Network,Springer, pp :1505-1511. https://doi.org/10.1007/s11276-015-1032-y

[9] M.Mohana Priya, Illango Krishnamurthi (2014), Modified DSR protocol for detection and removal of selective black hole attack in MANET, Computers and Electrical Engineering, Elsevier, vol 40, pp 530-538. https://doi.org/10.1016/j.compeleceng.2013.06.001

[10] Tao Shu, Marwan Krunz, (2015) Privacy-Preserving and Truthful Detection of Packet Dropping Attacks in Wireless Adhoc Networks, IEEE Transactions on Mobile Computing, 14(4), pp 813-828. https://doi.org/10.1109/tmc.2014.2330818

[11] Muhammad Saleem, Daniele Midi, Majid Iqbal khan, Elisa Bertino(2017),Fine Grained Analysis of Packet Loss in Manets", IEEE Access Multidisciplinary, vol 5, pp 7798-7806. https://doi.org/10.1109/access.2017.2694467

[12] M.Rajesh Babu, G.Usha (2016), A Novel Honey Pot Based Detection and Isolation Approach (NHBADI) to Detect and Isolate Black Hole Attacks in MANET", Wireless Personal Communication, Springer, and pp: 831-845, https://doi.org/10.1007/s11277-016$\underline{3229-5}$

[13] Djenouri D, Badache N(2008), Struggling Against Selfishness and Black Hole Attacks in MANETs, Wireless Communications \& Mobile Computing, 8(6), pp:689-704. https://doi. org/10.1002/wcm.493

[14] Jian Jian-MingChang,Po, Po-Chun Tsou, Isac Woungang, Han-Chieh Chao,and Chin-Feng Lai,(2015), Defending Against Collaborative Attacks by Malicious nodes in MANETs: A Cooperative Bait Detection Approach", IEEE Systems Journal,9(1),65-75. https://doi.org/ $10.1109 /$ jsyst.2013.2296197

[15] Raj.P.N, Swadas (2009) P.B: DPRAODV: A Dynamic Learning System against Blackhole Attack in AODV based MANET”, International Journal of Computer Science, vol 2, pp: 54-59. Doi: abs/0909.2371.

[16] Lauf AP, Peters RA, Robinson WH (2010),A Distributed Intrusion Detection System for Resource -constrained devices in adhoc Networks, Adhoc Network, Elsevier, 8(3), pp 253-266.https://doi.org/10.1016/j.adhoc.2009.08.002

[17] Nadeem A, Howarth MP,(2014),An Intrusion Detection and Adaptive Response Mechanism for MANET, Adhoc Networks, Elsevier, Vol 13, pp 368-380, , https://doi.org/ 10.1016/j.adhoc.2013.08.017.

[18] Rafsanjani MK, Aliahmadipour L, Javidi MM,(2010),An Optimal method for Detecting Internal and External Intrusion in MANETs, Communication and Networking, springer: Berlin/Heidelberg, pp 71-82. https://doi.org/10.1007/978-3-642-17604-3_8. 
[19] Weizhi Meng, WenJuan, Lam For Kwok, (2017) Towards Effective Filtering Trust Based Packet Filtering in Collaborative Network Environments, IEEE Transaction on Network and Service Management, 14(1),pp:233-245. https://doi.org/10.1109/tnsm.2017.2664893

[20] Jinfang Jiang ,Guangjie Han,Feng Wang,Lei Shu,Mohsen Guizani, (2015)An Efficient Distributed Trust Model for Wireless Sensor Networks",IEEE Transactions on Parallel and Distributed System,26(5),pp 1228-1237. https://doi.org/10.1109/tpds.2014.2320505

[21] Antesar M.Shabut, Keshav P.Dahal, Sanat Kumar Bista, Irfan U.Awan (2015), Recommendation Based Trust Model with an Effective Defense Scheme for Manets", IEEE Transactions on Mobile Computing, 14(10), pp 2101-2114. https://doi.org/10.1109/tmc.20 14.2374154

[22] Danyang Qin,Songxiang Yang, Shuang Jia,Yan Zhang,Jingya Ma And Qun Ding,(2017),Research on Trust sensing Based Secure Routing Mechanism for wireless Sensor Networks",IEEE Access Multidisciplinary, Vol 5, pp 9599-9609. https://doi.org/ 10.1109/access.2017.2706973.

[23] Vijender Busi Reddy, Sarma Venkataraman,Atul Negi, (2017) Communication and Data Trust for Wireless Sensor Networks Using D-S Theory",IEEE Sensors Journal,17(12),pp 3921-3928. https://doi.org/10.1109/jsen.2017.2699561

[24] Sha Shauaishuai Tan, Xiaoping Li and Qingkuan Dong, (2016),A Trust Management System for Securing Data Plane of Adhoc Networks, IEEE Transactions on Vehicular Technology, 65(9), 2016, pp 7579-7592. https://doi.org/10.1109/tvt.2015.2495325

\section{Authors}

M. Thebiga has received her Master degree in Software Engineering from periyar Maniammai college of Technology for Women, Thanjavur, TamilNadu, and pursuing her Ph.D. in Computer Science and Engineering from Noorul Islam Centre for Higher Education, Kumaracoil, and TamilNadu. Her research interest includes Mobile Adhoc Networks, Wireless networks and Security, Quality of Service

R. Suji Pramila is currently working as an Associate Professor in Noorul Islam Centre for Higher Education, Kumaracoil, and TamilNadu. She received her Ph.D. in Computer Science and Engineering from Noorul Islam Centre for Higher Education, Kumaracoil. Her research interests includes Mobile communication and Sensor Networks.

Article submitted 2019-07-18. Resubmitted 2019-09-24. Final acceptance 2019-09-30. Final version published as submitted by the authors. 